\section{ENDOSCOPIC TRANSANAL VACUUM-ASSISTED CLOSURE OF LOW PELVIC CAVITIES FOLLOWING ANASTOMOTIC AND STUMP LEAKAGE}

G Martel, K McElvanna, K McCallion, I McAllister Department of Colorectal Surgery, Ulster Hospital Dundonald, Belfast, Northern Ireland

10.1136/gutjnl-2013-305143.108

Introduction Endoscopic transanal vacuum-assisted closure is a minimally invasive method used in the treatment of low pelvic anastomotic leaks and symptomatic cavities.

Aims/Background We report our experience with this technique using Endo-Sponge therapy in a series of 10 patients.

Method All patients treated with Endo-Sponge therapy between November 2008 and January 2013 in our hospital were identified. Data collected retrospectively from clinical and endoscopic notes included indication, duration of therapy, number of sponge changes, complications and outcome.

Results Ten patients ( 8 male, median age 59 years) underwent Endo-Sponge therapy. Seven were treated for anastomotic leaks following low anterior resection. Two patients had symptomatic low pelvic cavities following ileal pouch excision and 1 was treated for a pelvic cavity following a perforated low Hartmann's stump. Seven patients were treated within 17 days (0-17 days) of diagnosis of pelvic sepsis. The remaining 3 patients were treated for chronic low pelvic cavities at intervals ranging from 5 months following index surgery. Median duration of treatment was 28.5 days ( $8-40$ days) with a median number of sponge changes of 7 (2-11 changes). One patient developed a transient inflammatory response and pneumoperitoneum which settled with antibiotic therapy but there were no other complications. All patients had clinical resolution of pelvic sepsis and four patients had definitive cavity closure.

Conclusion Endoscopic transanal vacuum-assisted closure is an effective low-risk method of controlling pelvic sepsis following anastomotic leak and can be used in the management of symptomatic cavities following pouch excision and low rectal stump leakage. 